RAIRO-Inf. Theor. Appl. 40 (2006) v-xiii

DOI: $10.1051 /$ ita:2006018

\title{
Preface
}

This special issue is a tribute to Alberto Bertoni on the occasion of his 60th birthday, July 17, 2006. It collects works from co-authors, close friends or former students and covers many branches of Theoretical Computer Science to which Alberto contributed. This is the way the editors of this issue express their deep conviction that this area would not be what it is, had Alberto chosen to do research in Physics as he was initially tempted.

\section{A SHORT BIOGRAPHY}

Alberto Bertoni, the first of five children, was born in Barlassina, a small town north of Milano, July 17, 1946. His father (1911-1996) was an accountant for a local bank. His mother, Innocente Rosa Borghi, born in 1926, was trained as a primary school teacher but worked only one year as such. She is the cousin of the late physicist Don Carlo Borghi, a catholic priest who elaborated an original theory of the hydrogen atom and anticipated some ideas and experiments connected with cold fusion. Alberto is married to Luciana Peverelli who teaches French in high school.

He attended the local primary school (the five first years) in Barlassina. For the three following years he was a pupil in the secondary school in the region of Lombardia. Finally he spent the last five years in the liceo scientifico "Vittorio Veneto" in Milano before becoming a student in Physics at the Università degli Studi di Milano. At that time, the curriculum consisted of four years which ended with the defense of a "laurea" thesis. Alberto hesitated between working with Professor Occhialini on gravitational waves or writing a thesis on the theory of games à la von Neumann. In the end he chose to work in the theory of fuzzy sets under the supervision of Professor Giovanni Degli Antoni. He graduated in Physics with the highest grade and cum laude, July 22, 1970. His thesis was entitled "Teoria degli insiemi fuzzy e sue applicazioni alla teoria degli automi" (The theory of fuzzy sets and its applications to the theory of automata).

In order to better grasp Alberto's professional life, a few words on the general situation of Computer Science in Italy in the seventies from both viewpoints of research and teaching might be useful. In most countries, Computer Science emancipated from Mathematics. In Italy, it often emerged from Physics and many Computer Scientists born before 1950 graduated in Physics. In the specific case of the University of Milano, Computer Science was given visibility inside the research

(c) EDP Sciences 2006 
structure of the Istituto di Fisica by the Gruppo di Elettronica e Cibernetica, then transformed in 1977 into the Istituto di Cibernetica, which formally accessed autonomy in 1986 as a full-fledged Dipartimento di Scienze dell'Informazione (Department of Information Sciences). Concerning teaching, the appointment for chairs in a given discipline was the result of a centralized process. A nationwide competition was organized and the winners were "called up" by the Universities to which the Ministry had allowed the opening of a position in that field. A curriculum in Computer Science was organized at the Università di Pisa in 1968 and for many years it remained the only one in Italy. An independent undergraduate course in Information Sciences was created at the University of Milano under the impulse of Professor Giovanni Degli Antoni in the academic year 1981-1982.

After his graduation, Alberto received a CNR fellowship for a couple of years, then a national research contract from the Ministry of Education. He also taught "Istituzioni di matematiche", an evening course of Mathematics in the Biology curriculum, as a lecturer under various statuses. In 1980 he competed for a position of full professor in Computer Science. He was listed among the winners and subsequently was called up by the University of Cosenza, Calabria. The next year, when the Università degli Studi di Milano obtained its degree in Computer Science he asked his transfer and moved back to Milano as a full professor.

\section{THE SCIENTIST}

Alberto contributed to the training of quite a number of researchers, through his advisory activity for over 150 degree theses ("lauree") in Computer Science, Mathematics and Physics and a score of PhD theses in Computer Science, Mathematics and Engineering. He sees his role not only as being available for interviews and sharing his knowledge on different scientific issues but also as giving handouts for all courses he teaches and occasionally writing monographs on emergent topics before they become fashionable. A personal experience of the French editor of this issue illustrates his gift as a lecturer. He met him for the first time in 1986 in a workshop held in Paris on the Theory of Traces. Alberto announced to the audience the well celebrated result of Wiesław Zielonka on asynchronous automata which he knew through a preliminary version. He was far from mastering French and yet he was able to provide a perfectly clear and intelligible explanation, quite an achievement. All those who have listened to his lectures or talks were struck by his great capacity of extracting the core of a topic. More generally, he never leaves unanswered a technical question, even when the person lacks the background, but he never simplifies to the point that his speech would be misleading.

Concerning research, one has to bear in mind that Alberto would have been a brilliant physicist had he not lived in the era of Informatics. The precise survey he wrote at the occasion of the event in honor of his relative Don Carlo Borghi shows how deep remains his understanding of the main issues that were at stake in Physics in Italy during the period 1930-1960. Physics is faced with the necessity of providing a coherent theory from experimental data subject to errors. A physicist must therefore abstract away from the measurements so as to go beyond apparent inconsistencies. In the language of Computer Science, the approach must 
be "fault tolerant". This requires intuition and capacity of abstraction. A more formal attitude which would try at any cost to design a theory which would match exactly all the data would probably be deemed to fail. The Computer Scientist is faced with similar difficulties. Alberto applies this skill in order to get correct statements for the property he investigates but at the same time he has the rigor of the mathematician when it comes to prove them. He is extremely versatile and has contributed to many areas such as formal languages and its extensions to trace theory and formal series with applications to random generation and counting problems, computational learning including neural networks and genetic models with applications to combinatorial optimization problems, complexity theory in the models of probabilistic and quantum machines as well as in various models of parallel computation.

\section{THE ORGANIZER}

At the local level, Alberto is greatly involved with the future developement of his University and participates actively in the different steps of the construction of the new campus. This relatively unrewarding activity is typical of Alberto's generosity and readiness to work for the good of his colleagues, students, university and more generally of his country without expecting a personal benefit.

He was co-promoter of the Italian Association of Theoretical Computer Science (Italian Chapter of EATCS), first President of this association for six years (198794) and Italian member in the Council of the European Association of Theoretical Computer Science. He is a member of the Editorial Board of Theoretical Informatics and Applications and has been a member of the Program Committee of several national and international conferences.

Paris, Milano, June 2006

PAOLA CAMPADELLI Christian Choffrut

Massimiliano GoldwURm MAURO TORELli 


\section{BIBLIOGRAPHY}

\section{REFERENCES}

[1] M.A. Alberti, A. Bertoni, P. Campadelli, G. Grossi and R. Posenato, A neural circuit for the maximum 2-satisfiability problem, in 3rd Euromicro Workshop on Parallel and Distributed Processing (PDP '95), IEEE Computer Society (1995) 319-323.

[2] M.A. Alberti, A. Bertoni, P. Campadelli, G. Grossi and R. Posenato, A neural algorithm for max-2sat: Performance analysis and circuit implementation. Neural Networks 10 (1997) $555-560$.

[3] M. Anselmo and A. Bertoni, Two-way probabilistic automata and rational power series, in Proceedings of the Fourth Italian Conference on Theoretical Computer Science. World Scientific (1992) 9-23.

[4] B. Apolloni, A. Bertoni, P. Campadelli and D. de Falco, Neural networks: Deterministic and stochastic dynamics, in Proceedings of a Workshop on Dynamics and Stochastic Processes. Lect. Notes Phys. 335 (1989) 27-41.

[5] B. Apolloni, A. Bertoni, P. Campadelli and D. de Falco, Binary networks with parallel updating, in Proceedings of the 3rd Italian Workshop on Parallel Architectures and Neural Networks, edited by E.R. Caianiello. World Scientific (1990) 47-56.

[6] B. Apolloni, A. Bertoni, P. Campadelli and D. de Falco, Asymmetric boltzmann machines. Biological Cybernetic 66 (1991) 61-70.

[7] B. Apolloni, A. Bertoni, P. Campadelli and D. De Falco, Lyapunov functions and neural networks, in Parallel Architectures and Neural Networks. Proceedings of the Second Italian Workshop, edited by E.R. Caianiello. World Scientific (1990) 53-67.

[8] B. Apolloni, A. Bertoni, P. Campadelli and G. Mauri, Formal models of learning from examples, in Proceedings of the 5th Italian Workshop on Parallel Architectures and Neural Networks, edited by E.R. Caianiello. World Scientific (1992) 47-76.

[9] A. Bertoni, P. Campadelli and G. Grossi, A discrete neural algorithm for the maximum clique problem: Analysis and circuit implementation, in Workshop on Algorithm Engineering (WAE'97), edited by S. Orlando Giuseppe and F. Italiano (1997) 84-91.

[10] A. Bertoni, P. Campadelli and G. Grossi, An approximation algorithm for the Maximum Cut problem and its experimental analysis, in Workshop on Algorithms and Experiments (ALEX'98), edited by R. Battiti and A. Bertossi (1998).

[11] A. Bertoni, Automi pesati ad interpretazione massimale, in Atti del Congresso di Cibernetica, edited by M.A. Baldocchi and F. Lenci. Felici (1971) 655-672.

[12] A. Bertoni, Complexity problems related to the approximation of probabilistic languages and events by deterministic machines, in ICALP, Automata, Languages and Programming, Colloquium, Paris, edited by M. Nivat. North-Holland (1972) 507-516.

[13] A. Bertoni, Equations of formal power series over non commutative semiring, in MFCS, Mathematical Foundations of Computer Science: Proceedings of Symposium and Summer School, Strbské Pleso (1973) 185-190.

[14] A. Bertoni, Grammatiche context-free su spazi metrici compatti, in Atti del Convegno AICA di Informatica Teorica, Editrice Tecnico Scientifica (1973) 327-351.

[15] A. Bertoni, Grammatiche pesate ed applicazioni, in Atti del Convegno AICA di Informatica Teorica, Editrice Tecnico Scientifica (1974) 117-137.

[16] A. Bertoni, Mathematical methods of the theory of stochastic automata, in MFCS, Mathematical Foundations of Computer Science, 3rd Symposium, edited by A. Blikle. Springer, Lect. Notes Comput. Sci. 28 (1974) 9-22.

[17] A. Bertoni, The solution of problems relative to probabilistic automata in the frame of the formal languages theory, in GI - 4. Jahrestagung, Berlin, edited by D. Siefkes. Springer, Lect. Notes Comput. Sci. 26 (1974) 107-112.

[18] A. Bertoni, Model theoretic aspects of abstract data specification, in Mathematical Logic in Computer Science, edited by B. Dömölki and T. Gergely. North-Holland (1981) 181-193. 
[19] A. Bertoni, M.C. Bollina, G. Mauri and N. Sabadini, On characterizing classes of efficiently parallelizable problems, in VLSI: Algorithms and Architectures, edited by P. Bertolazzi and F. Luccio. North-Holland (1985) 13-26.

[20] A. Bertoni, M. Brambilla, G. Mauri and N. Sabadini, An application of the theory of free partially commutative monoids: Asymptotic densities of trace languages, in Mathematical Foundations of Computer Science 1981, edited by J. Gruska and M. Chytil. Springer, Lect. Notes Comput. Sci. 118 (1981) 205-215.

[21] A. Bertoni, R. Brivio and P. Campadelli, Stabilization and size of attraction basins in symmetric networks, in Parallel Architectures and Neural Networks. Proceedings of the Fourth Italian Workshop, edited by E.R. Caianiello. World Scientific (1991) 281-284.

[22] A. Bertoni, D. Bruschi and M. Goldwurm, Ranking and formal power series. Theor. Comput. Sci. 79 (1991) 25-35.

[23] A. Bertoni, D. Bruschi, D. Joseph, M. Sitharam and P. Young, Generalized Boolean hierarchies and Boolean hierarchies over RP (Conference Abstract), in Fundamentals of Computation Theory, International Conference FCT'89, edited by J. Csirik, J. Demetrovics and F. Gécseg. Springer, Lect. Notes Comput. Sci. 380 (1989) 35-46.

[24] A. Bertoni and P. Campadelli, Neural networks and non-uniform circuits, in Parallel Architectures and Neural Networks, in Proceedings of the First Italian Workshop, edited by E.R. Caianiello. World Scientific (1989) 18-27.

[25] A. Bertoni and P. Campadelli, Analysis of parallel and sequential Boltzmann machines, in Proceedings of the International Neural Networks Conference, Parigi (1990).

[26] A. Bertoni and P. Campadelli, Formal models of learning from examples, in Proceedings of ICANN94. Springer-Verlag (1992) 1157-1160.

[27] A. Bertoni, P. Campadelli, M. Carpentieri and G. Grossi, A genetic model and the Hopfield networks, in Artificial Neural Networks - ICANN 96, edited by C. von der Malsburg, W. von Seelen, J.C. Vorbrüggen and B. Sendhoff. Springer, Lect. Notes Comput. Sci. 1112 (1996) 463-468.

[28] A. Bertoni, P. Campadelli, M. Carpentieri and G. Grossi, Analysis of a genetic model, in Proceedings of the 7th International Conference on Genetic Algorithms, East Lansing, MI, USA, July 19-23, 1997, edited by T. Bäck. Morgan Kaufmann (1997) 121-126.

[29] A. Bertoni, P. Campadelli, M. Carpentieri and G. Grossi, A genetic model: Analysis and application to maxsat. Evol. Comput. 8 (2000) 291-309.

[30] A. Bertoni, P. Campadelli and N. Cesa-Bianchi, Multilayer perceptrons and learning, in Proceedings of the 5th Italian Conference on Theoretical Computer Science, edited by A. De Santis. World Scientific (1996) 43-76.

[31] A. Bertoni, P. Campadelli, C. Gangai and R. Posenato, Approximability of the ground state problem for certain Ising spin glasses. J. Complexity 13 (1997) 326-329.

[32] A. Bertoni, P. Campadelli and F. Grassani, Full parallelism in Boltzmann machines, in Proceedings of NeuroNimes89: International Workshop on Neural Networks and their Applications (1989) 361-370.

[33] A. Bertoni, P. Campadelli and G. Grossi, An approximation algorithm for the maximum cut problem and its experimental analysis. Discrete Appl. Math. 110 (2001) 3-12.

[34] A. Bertoni, P. Campadelli and G. Grossi, A neural algorithm for the maximum clique problem: Analysis, experiments, and circuit implementation. Algorithmica 33 (2002) 71-88.

[35] A. Bertoni, P. Campadelli and G. Grossi, Solving min vertex cover with iterated Hopfield networks, in Proceedings of the 12th Italian Workshop on Neural Nets, edited by R. Tagliaferri and M. Marinaro. Springer (2002) 87-95.

[36] A. Bertoni, P. Campadelli and A. Manfredi, Robust learning by means of single neurons, in Neural Nets, WIRN Vietri '93: Sixth Italian Workshop, edited by E.R. Caianiello. World Scientific (1994) 192-198.

[37] A. Bertoni, P. Campadelli and G. Mauri, Some notes on computational learning theory. Bulletin of the EATCS 43 (1991) 140-158. 
[38] A. Bertoni, P. Campadelli and G. Mauri, PAC learning and neural networks, in Proceedings of the Conference on Structure from Physics to General System, edited by G. Scarpetta and M. Marinaro. World Scientific (1992) 48-63.

[39] A. Bertoni, P. Campadelli and G. Molteni, On the approximability of the energy function of Ising spin glasses. J. Phys. A: Math. Gen. 27 (1994) 6719-6729.

[40] A. Bertoni, P. Campadelli and A. Morpurgo, Total stabilization in symmetric networks, in Proceedings of NeuroNimes88: International Workshop on Neural Networks and their Applications (1988) 183-197.

[41] A. Bertoni, P. Campadelli, A. Morpurgo and S. Panizza, Polynomial uniform convergence of relative frequencies to probabilities, in Advances in Neural Information Processing Systems 4, NIPS, edited by J.E. Moody, S.J. Hanson and R. Lippmann. Morgan Kaufmann (1991) $904-911$.

[42] A. Bertoni, P. Campadelli, A. Morpurgo and S. Panizza, Polynomial uniform convergence and polynomial-sample learnability, in COLT (1992) 265-271.

[43] A. Bertoni, P. Campadelli, A. Morpurgo and R. Posenato, An algorithm for learning from positive examples classes of linearly separable Boolean functions, in Parallel Architectures and Neural Networks, in Proceedings of the Fourth Italian Workshop, edited by E.R. Caianiello. World Scientific (1991) 11-19.

[44] A. Bertoni, P. Campadelli, A. Morpurgo and R. Posenato, An algorithm for learning from positive examples classes of linearly separable Boolean functions, in Proceedings of the 4th Italian Workshop on Parallel Architectures and Neural Networks, edited by E.R. Caianiello. World Scientific (1991) 11-19.

[45] A. Bertoni, P. Campadelli and S. Panizza, Learnability by fixed distributions: An information theoretic approach, in Proceedings of the Fourth Italian Conference on Theoretical Computer Science. World Scientific (1992) 68-79.

[46] A. Bertoni, P. Campadelli and M. Parodi, A boosting algorithm for regression, in Artificial Neural Networks - ICANN '97, edited by W. Gerstner, A. Germond, M. Hasler and J.-D. Nicoud. Springer, Lect. Notes Comput. Sci. 1327 (1997) 343-348.

[47] A. Bertoni, P. Campadelli and R. Posenato, Polynomial time approximation of min-energy in Hopfield networks, in Proceedings of the 7th Italian Workshop on Parallel Architectures and Neural Networks, edited by R. Tagliaferri and M. Marinaro. World Scientific (1995) $165-170$.

[48] A. Bertoni, P. Campadelli and R. Posenato, An upper bound for the maximum cut mean value, in Graph-Theoretic Concepts in Computer Science, 23rd International Workshop, WG '97, edited by R.H. Möhring. Springer, Lect. Notes Comput. Sci. 1335 (1997) 78-84.

[49] A. Bertoni, P. Campadelli and R. Posenato, Analysis of a genetic model with finite populations, in Advances in Natural Computation, First International Conference, ICNC 2005, Changsha, China, August 27-29, 2005, Proceedings, Part III, edited by L. Wang, K. Chen and Y.-S. Ong. Springer, Lect. Notes Comput. Sci. 3612 (2005) 235-244.

[50] A. Bertoni, P. Campadelli, R. Posenato and M. Santini, Approximability of ground state problem on tridimensional Ising spin glasses, in Proceedings of the 5th Italian Conference on Theoretical Computer Science, edited by A. De Santis. World Scientific (1996) 492-496.

[51] A. Bertoni and M. Carpentieri, Analogies and differences between quantum and stochastic automata. Theor. Comput. Sci. 262 (2001) 69-81.

[52] A. Bertoni and M. Carpentieri, Regular languages accepted by quantum automata. Inf. Comput. 165 (2001) 174-182.

[53] A. Bertoni, N. Cesa-Bianchi and G. Fiorino, Efficient learning with equivalence queries of conjunctions of modulo functions. Inf. Process. Lett. 56 (1995) 15-17.

[54] A. Bertoni, C. Choffrut, M. Goldwurm and V. Lonati, On the number of occurrences of a symbol in words of regular languages. Theor. Comput. Sci. 1-3 (2003) 431-456.

[55] A. Bertoni, C. Choffrut, M. Goldwurm and V. Lonati, Local limit distributions in pattern statistics: Beyond the markovian models, in STACS 2004, 21st Annual Symposium on Theoretical Aspects of Computer Science, edited by V. Diekert and M. Habib. Lect. Notes Comput. Sci. 2996 (2004) 117-128. 
[56] A. Bertoni, C. Choffrut, M. Goldwurm and V. Lonati, Local limit properties for pattern statistics and rational models. Theory Comput. Syst. 39 (2006) 209-235.

[57] A. Bertoni and M. Cugiani, Sulla natura dell'insieme di una copertura dei punti razionali, Rend. Sci., Istituto Lombardo, Accademia di Scienze e Lettere A 127 (1994) 227-232.

[58] A. Bertoni and M. Dorigo, Implicit parallelism in genetic algorithms. Artif. Intell. 61 (1993) 307-314.

[59] A. Bertoni, R. Folgieri and G. Valentini, Feature selection combined with random subspace ensemble for gene expression based diagnosis of malignancies, in 15th Italian Workshop on Neural Nets, WIRN 2004, Università di Perugia (2004).

[60] A. Bertoni, R. Folgieri and G. Valentini, Biomolecular cancer prediction with random subspaces ensembles of support vector machines. Neurocomputing 63 (2005) 535-539.

[61] A. Bertoni and M. Goldwurm, On the prefixes of a random trace and the membership problem for context-free trace languages, in Applied Algebra, Algebraic Algorithms and Error-Correcting Codes, 5th International Conference, $A A E C C-5$, edited by L. Huguet and A. Poli. Springer, Lect. Notes Comput. Sci. 356 (1987) 35-59.

[62] A. Bertoni and M. Goldwurm, On ranking 1-way finitely ambiguous NL languages and \#P $\mathrm{P}_{1}$-complete census functions. RAIRO-Inf. Theor. Appl. 27 (1993) 135-148.

[63] A. Bertoni, M. Goldwurm and P. Massazza, Counting problems and algebraic formal power series in noncommuting variables. Inf. Process. Lett. 34 (1990) 117-121.

[64] A. Bertoni, M. Goldwurm, G. Mauri and N. Sabadini, Parallel algorithms and the classification of problems, in WOPPLOT 86 - Parallel Processing: Logic, Organization, and Technology, edited by J.D. Becker and I. Eisele. Springer, Lect. Notes Comput. Sci. 253 (1986) 206-226.

[65] A. Bertoni, M. Goldwurm, G. Mauri and N. Sabadini, Counting techniques for inclusion, equivalence and membership problems. Chapter 5, in The Book of Traces, edited by V. Diekert and G. Rozenberg. World Scientific (1995) 131-164.

[66] A. Bertoni, M. Goldwurm and N. Sabadini, Analysis of a class of algorithms for problems on trace languages, in Applied Algebra, Algebraic Algorithms and Error-Correcting Codes, 4 th International Conference, $A A E C C$-4, edited by T. Beth and M. Clausen. Springer, Lect. Notes Comput. Sci. 307 (1986) 202-214.

[67] A. Bertoni, M. Goldwurm and N. Sabadini, Computing the counting function of contextfree languages, in STACS 8\%, 4th Annual Symposium on Theoretical Aspects of Computer Science, edited by F.-J. Brandenburg, G. Vidal-Naquet and M. Wirsing. Lect. Notes Comput. Sci. 247 (1987) 169-179.

[68] A. Bertoni, M. Goldwurm and N. Sabadini, The complexity of computing the number of strings of given length in context-free languages. Theor. Comput. Sci. 86 (1991) 325-342.

[69] A. Bertoni, M. Goldwurm and M. Santini, Random generation and approximate counting of ambiguously described combinatorial structures, in STACS 2000, edited by H. Reichel and S. Tison. Springer, Lect. Notes Comput. Sci. 1770 (2000) 567-580.

[70] A. Bertoni, M. Goldwurm and M. Santini, Random generation for finitely ambiguous context-free languages. RAIRO-Inf. Theor. Appl. 35 (2001) 499-512.

[71] A. Bertoni, G. Haus, G. Mauri and M. Torelli, Compattazione di strutture informative nella descrizione di processi musicali, in Atti del Congresso Annuale AICA. Dedalo (1976) 497-508.

[72] A. Bertoni and P. Massazza, On the inclusion problem for finitely ambiguous rational trace languages. RAIRO-Inf. Theor. Appl. 32 (1998) 79-98.

[73] A. Bertoni, P. Massazza and R. Radicioni, Random generation of words with fixed occurences in regular languages, in WORDS'03, 4th International Conference on Combinatorics on Words. September 2003, Turku, Finland (2003) 332-343.

[74] A. Bertoni, P. Massazza and N. Sabadini, Holonomic generating functions and context free languages. Int. J. Found. Comput. Sci. 3 (1992) 181-191.

[75] A. Bertoni and G. Mauri, Aspetti algebrico-categoriali di problemi combinatori, in Atti del Congresso Annuale AICA (1975) 387-393. 
[76] A. Bertoni and G. Mauri, Introduzione alle serie formali, Quaderni di Informatica Teorica, ISEDI 2 (1975).

[77] A. Bertoni and G. Mauri, Il linguaggio delle categorie nell'informatica teorica, Quaderni di Informatica Teorica. ISEDI 4 (1977).

[78] A. Bertoni and G. Mauri, On efficient computation of the coefficients of some polynomials with applications to some enumeration problems. Inf. Process. Lett. 12 (1981) 142-145.

[79] A. Bertoni, G. Mauri and P. Miglioli, Una guida euristica alla soluzione di problemi combinatori, in Atti del Convegno Complessità di Calcolo, Modelli Gestionali e Territorio, CNR (1977) 130-145.

[80] A. Bertoni, G. Mauri and P. Miglioli, A characterization of abstract data as model-theoretic invariants, in Automata, Languages and Programming, 6th Colloquium, edited by H.A. Maurer. Springer, Lect. Notes Comput. Sci. 71 (1979) 26-37.

[81] A. Bertoni, G. Mauri and P. Miglioli, Towards a theory of abstract data types: A discussion on problems and tools, in International Symposium on Programming, Proceedings of the Fourth 'Colloque International sur la Programmation', edited by B. Robinet. Springer, Lect. Notes Comput. Sci. 83 (1980) 44-58.

[82] A. Bertoni, G. Mauri and P. Miglioli, On the power of model theory in specifying abstract data types and in capturing their recursiveness. Fundamenta Informaticae 6 (1983) 127170 .

[83] A. Bertoni, G. Mauri and P. Miglioli, Abstract data types and their extensions within a constructive logic, in Semantics of Data Types, International Symposium, edited by G. Kahn, D.B. MacQueen and G.D. Plotkin. Springer, Lect. Notes Comput. Sci. 173 (1984) 177-195.

[84] A. Bertoni, G. Mauri and N. Sabadini, A characterization of the class of functions computable in polynomial time on random access machines, in Conference Proceedings of the Thirteenth Annual ACM Symposium on Theory of Computation. ACM (1981) 168-176.

[85] A. Bertoni, G. Mauri and N. Sabadini, Disegno di programmi guidato dalle strutture di ingresso-uscita: un inquadramento teorico, in Atti Congresso Annuale AICA 2 (1981) $663-669$.

[86] A. Bertoni, G. Mauri and N. Sabadini, Context free trace languages, in CAAP'82 (1982) $32-42$.

[87] A. Bertoni, G. Mauri and N. Sabadini, Equivalence and membership problems for regular trace languages, in Automata, Languages and Programming, 9th Colloquium, edited by M. Nielsen and E.M. Schmidt. Springer, Lect. Notes Comput. Sci. 140 (1982) 61-71.

[88] A. Bertoni, G. Mauri and N. Sabadini, Metodologie di disegno di programmi guidate dalle strutture di dati di ingresso-uscita: Analisi formale di una proposta di estensione, in Atti Congresso Annuale AICA. CLEUP 1 (1982) 259-262.

[89] A. Bertoni, G. Mauri and N. Sabadini, Una classe di problemi vantaggiosamente parallelizzabili, in Atti del Congresso Annuale AICA, Liguori 1 (1983) 375-386.

[90] A. Bertoni, G. Mauri and N. Sabadini, Simulations among classes of random access machines and equivalence among numbers succintly represented, in Analysis and Design of Algorithms for Combinatorial Problems, edited by G. Ausiello and M. Lucertini. NorthHolland (1985) 65-89.

[91] A. Bertoni, G. Mauri and N. Sabadini, Membership problems for regular and context-free trace languages. Inf. Comput. 82 (1989) 135-150.

[92] A. Bertoni, G. Mauri and M. Torelli, An algebraic approach to problem solution and problem semantics, in Mathematical Foundations of Computer Science 1977, 6th Symposium, edited by J. Gruska. Springer, Lect. Notes Comput. Sci. 53 (1977) 253-262.

[93] A. Bertoni, G. Mauri and M. Torelli, Some recursive unsolvable problems relating to isolated cutpoints in probabilistic automata, in Automata, Languages and Programming, Fourth Colloquium, edited by A. Salomaa and M. Steinby. Springer, Lect. Notes Comput. Sci. 52 (1977) 87-94.

[94] A. Bertoni, G. Mauri and M. Torelli, Three efficient algorithms for counting problems. Inf. Process. Lett. 8 (1979) 50-53. 
[95] A. Bertoni, C. Mereghetti and B. Palano, Approximating stochastic events by quantum automata, in ERATO International Conference on Quantum Information Science 2003, Kyoto, Japan (2003).

[96] A. Bertoni, C. Mereghetti and B. Palano, Golomb rulers and difference sets for succinct quantum automata. Int. J. Found. Comput. Sci. 14 (2003) 871-888.

[97] A. Bertoni, C. Mereghetti and B. Palano, Lower bounds on the size of quantum automata accepting unary languages, in Theoretical Computer Science, 8th Italian Conference, ICTCS 2003, Bertinoro, Italy, October 13-15, 2003, Proceedings, edited by C. Blundo and C. Laneve. Springer, Lect. Notes Comput. Sci. 2841 (2003) 86-96.

[98] A. Bertoni, C. Mereghetti and B. Palano, Quantum computing: 1-way quantum automata, in Developments in Language Theory, 7th International Conference, DLT 2003, Szeged, Hungary, July 7-11, 2003, Proceedings, edited by Z. Ésik and Z. Fülöp. Springer, Lect. Notes Comput. Sci. 2710 (2003) 1-20.

[99] A. Bertoni, C. Mereghetti and B. Palano, Small size quantum automata recognizing some regular languages. Theor. Comput. Sci. 340 (2005) 394-407.

[100] A. Bertoni, C. Mereghetti and B. Palano, Some formal methods for analyzing quantum automata, in 7th International Workshop on Descriptional Complexity of Formal Systems (DCFS'05), Como (2005) 1-14.

[101] A. Bertoni, C. Mereghetti and G. Pighizzini, Corrigendum: An optimal lower bound for nonregular languages. Inf. Process. Lett. 52 (1994) 339.

[102] A. Bertoni, C. Mereghetti and G. Pighizzini, On languages accepted with simultaneous complexity bounds and their ranking problem, in Mathematical Foundations of Computer Science 1994, 19th International Symposium, MFCS'94, edited by I. Prívara, B. Rovan and P. Ruzicka. Springer, Lect. Notes Comput. Sci. 841 (1994) 245-255.

[103] A. Bertoni, C. Mereghetti and G. Pighizzini, An optimal lower bound for nonregular languages. Inf. Process. Lett. 50 (1994) 289-292.

[104] A. Bertoni, C. Mereghetti and G. Pighizzini, Strong optimal lower bounds for Turing machines that accept nonregular languages, in Mathematical Foundations of Computer Science 1995, 20th International Symposium, MFCS'95, edited by J. Wiedermann and P. Hájek. Springer, Lect. Notes Comput. Sci. 969 (1995) 309-318.

[105] A. Bertoni and B. Palano, Structural complexity and neural networks, in Neural Nets, 13th Italian Workshop on Neural Nets, WIRN, edited by M. Marinaro and R. Tagliaferri. Springer, Lect. Notes Comput. Sci. 2486 (2002) 190-215.

[106] A. Bertoni and N. Sabadini, Generating functions of trace languages. Bulletin of the EATCS 35 (1988) 106-112.

[107] A. Bertoni and M. Torelli, Elementi di matematica combinatoria, Serie di informatica. ISEDI 4 (1977).

[108] A. Bertoni and G. Valentini, Ensembles based on random projections to improve the accuracy of clustering algorithms, in 16th Italian Workshop on Neural Nets, WIRN, 2005. Springer, Lect. Notes Comput. Sci. 3931 (2006) 31-37.

[109] N. Sabadini, G. Pighizzini, G. Mauri and A. Bertoni, Algebraic and informational aspects of Zielonka's theorem, in Semigroups: Algebraic Theory and Applications to Formal Languages and Codes, edited by C. Tibiletti, C. Bonzini and A. Cherubini. World Scientific (1993) 17-26. 